\title{
BCG vaccine and pulmonary tuberculosis
}

\author{
Tafreshi SH* \\ R\&D Department, Pasteur Institute of Iran, Alborz, Iran.
}

\begin{abstract}
Tuberculosis (TB) is one of the most important public health problems in the world and has been declared as a global emergency by the World Health Organization (WHO) in 1993. Bacillus Calmette-Guérin (BCG) is one of the first developed vaccines to prevent TB. Unfortunately, BCG vaccine has had a limited duration of protection and has shown controversial and variable levels of efficacy, especially against pulmonary TB. On the other hand, efforts in production of new forms of this vaccine have been in vain and revaccination is not considered as a suitable strategy to control the disease. Considering that TB affects adults mostly between the ages of 15 and 59, pulmonary TB is more common than disseminated TB and BCG vaccine has had a minimal effect on the global burden of pulmonary TB. Before introducing a new, safe and effective vaccine, combined strategies in the field of control and treatment of TB rather than vaccination are necessary.
\end{abstract}

KEYWORDS: BCG, pulmonary TB, revaccination, efficacy, prevention.

\section{INTRODUCTION}

Although it was believed in 2002 that tuberculosis (TB) is under control or at least controllable in high income countries[1], the prevalence of $\mathrm{TB}$ has been increasing for reasons including:1) poverty and severe socio-economic class differences between the rich and the poor population groups in various communities (not only in the developing countries but also in certain groups of the developed countries), 2) demographic changes such as the increasing world population, changing population age pyramid, migration, poor hygiene in countries experiencing severe economic crisis and civil strife, 3) the global HIV/AIDS pandemic, 4) drug or alcohol abuse and malnutrition [2-3] and 5) challenges in the diagnosis and treatment of TB [4-5]. For these reasons, in 1993, the World Health Organization (WHO) declared the TB epidemic as a global public health emergency [1]. In 2014, 9.6 million new cases of TB and 1.5 million deaths (1.1 million among the HIVnegative people and 0.4 million among the HIV-positive people) were estimated by WHO. Globally, $3.3 \%$ of new cases and $20 \%$ of previously-treated TB patients were infected with multidrug-resistant strains of Mycobacterium tuberculosis (MDR-TB) [6]. A review of the current studies shows that the effectiveness of BCG vaccine depends on several factors while the vaccine is not efficient in the management of pulmonary $\underline{T B}$, especially in adults and new vaccines are needed in order

*Corresponding Author: Seyed Hesameddin Tafreshi, Research and Production Complex R\&D Department, Pasteur Institute of Iran, 25th km Tehran-Karaj Highway 31599-15111.

Email: tafreshi@pasteur.ac.ir

Tel/Fax: (+98) 2636100965/ (+98) 2636102900 to control this disease. This paper has reviewed these factors which affect the efficacy of the vaccine and has emphasized on the necessity of special attention to other factors affecting the control and prevention of the disease until the development of new and effective vaccines.

\section{MATERIALS and METHODS}

For each subject, a review of electronic sources was carried out on PubMed and Google Scholar using appropriate key words such as BCG vaccine, indication, efficacy, pulmonary tuberculosis, prevention and control.

Tuberculosis prevention strategies

TB can be prevented by the following strategies:

1- Primary prevention strategies: These strategies are aimed to prevent new cases of TB infection by means of:

- Vaccination

- Preventive therapy

- Environmental controls such as ventilation and indirect ultraviolet irradiation (implemented only in hospitals)

2- Secondary prevention strategies: aimed to prevent TB disease and to block the progression of TB infection to the active form of the disease or to reduce the spreading of the infection in the population by means of:

- Early diagnosis of TB

- Rapid finding of the active cases 
- Screening programs for the detection of active TB patients

- Identification and testing of the high-risk groups such as healthcare workers, homeless persons, immunocompromised individuals, people traveling or living in the endemic areas, people living in the institutions (e.g. nursing homes, prisons, shelters, schools, child care centers, rehabilitation facilities and treatment facilities)

- Isolation and quarantining of the suspected individuals

- Contact investigation by healthcare workers in order to identify people who may have been exposed to the active patients

- Treatment of latent TB infection

- Treatment of active disease cases

- Treatment of HIV co-infection cases and prevention of mixing of $\mathrm{TB}$ and HIV patients in healthcare centers

- Treatment of drug resistant TB

- Education of healthcare workers for quick detection and prompt referral to medical services as well as the population about signs and symptoms of the disease in order to reduce the risk of transmission to others and seeking medical care

All practices within these strategies must be performed according to approved and written policies and protocols of a comprehensive TB management program [7-12]. Obviously, among these strategies, vaccination which plays a major role in the prevention and control of TB should be considered as the main focus.

\section{History and applications of BCG}

BCG, named after the family names of Albert Calmette and Camille Guérin) is attenuated live bovine tuberculosis bacillus, Mycobacterium bovis which was developed in 1919 and has been used for humans since 1921. BCG vaccine is mostly prepared from three strains of Pasteur 1173P2, Danish 1331 and Tokyo 172 [13-15]. The vaccine is basically used for prevention of $\mathrm{TB}$; however other applications have been reported for it, including:

- Protection against leprosy [16-17]

- Protection or delay in Buruli ulcer [18]

- Immunotherapy of cancers such as bladder cancer [19], colorectal cancer [20], lung cancer [21] malignant peripheral nerve sheath tumor [22] and melanoma [23]

- Diseases such as multiple sclerosis [24-26] and Parkinson's [27-29] in animal models

\section{The efficacy of BCG}

The main intended use of BCG is prevention of TB. However unfortunately, after more than 90 years of the administration of the vaccine, the investigations point out that the efficacy of the vaccine has shown different outcomes. For example, this vaccine prevents serious types of the disease in children such as meningitis TB and miliary TB; however, its efficacy in adults has been highly variable. Meanwhile, older people who are most likely to spread the disease and have the highest risk of death from TB remain unprotected $[6,29]$. The efficacy of BCG vaccine has been varied from 0 to $80 \%$ in different populations of the world [30-31]. For example, the results of two meta-analysis studies have shown that vaccination with BCG has decreased the risk of TB in all types of the disease $(\sim 50 \%)$ [32] as well as meningitis and miliary forms (excluding the pulmonary TB)for at least $70 \%$ in children [33].
Interestingly, a follow-up study has revealed the complete lack of protective efficacy against pulmonary TB in adults [34-35]. In terms of duration of the protection, BCG vaccine protects children for about 10 years [36]; however, there is a limited evidence for the periods of more than 10 years. For instance, a study looking at Native Americans vaccinated with BCG in the 1930 s, has found evidence of the protection even 50 to 60 years after the immunization [37]. On the other hand, BCG revaccination has not provided additional protection [38]. Principally, revaccination is not considered as a strategy to control the disease [39].

Why is the vaccine efficacy variable?

A number of possible reasons for the variable efficacy of BCG in different countries can be summarized as:

- The type of culture medium[30] and the differences in BCG vaccine strains [40]

- The route of administration [41]

- Genetic variation in the studied populations [42]

- The masking effect: It happens when exposure to environmental mycobacteria such as M. avium, $M$. marinum and $M$. intracellular that causes nonspecific immune responses against mycobacteria while BCG does not induce additional specific immune responses. This effect is called masking, because the protection effect of BCG is masked by the previous non-specific immune responses against environmental mycobacteria. This effect has been shown in a study of school children in England and Malawi [43-44].

- Blocking hypothesis: According to this hypothesis that has been studied in mice, the immune responses against environmental mycobacteria can prevent the BCG proliferation in the vaccinee and thus the optimal immune response is not achieved with BCG vaccination [45].

- Parasitic co-infections: Simultaneous infection with certain parasitic diseases can change the immune response from Th1 (the response required to prevent TB disease) to Th2 and therefore BCG vaccine loses its efficacy [46].

- The effect of ultraviolet radiation: It has been shown that ultraviolet radiation type B (UVB) reduces the vaccine efficacy in guinea pigs [47].

- Oxidant-dependent immune responses: This hypothesis states that reducing BCG antioxidants enhances the immunogenicity and the balance between the host-generated oxidants and the microbial antioxidants affects the risk of developing pulmonary TB [14].

Necessity and approach to produce new vaccines

The WHO [6] and the US Centers for Disease Control and Prevention (CDC) have emphasized on the necessity of developing new vaccines to replace BCG against infection of M. tuberculosis [48]. Unfortunately, efforts in this field have been in vain yet. Different strategies have been considered to replace $\mathrm{BCG}$ vaccine including the application of the live whole cell, inactivated the whole cell as well as DNA and subunit vaccines [49]. Among these strategies, two of them using live vaccines and subunit vaccines are of the most interest [50].

In genetic manipulation, pathogenic genes are eliminated, or new genes are added to $M$. bovis in order to enhance the vaccine efficacy such as producing BCG without urease that presents more mycobacterial antigens to Tcell receptor. In the 
subunit vaccines, instead of the entire bacterium, particular antigens or epitopes (the specific parts of antigens that best stimulate the immune system) are recombinantly expressed and used as vaccines [51]. Different categories of vaccine candidates are summarized at Table 1 [52].

Table 1. Category and development phase of TB vaccine candidates

A) Viral vectored booster vaccines

\begin{tabular}{|c|c|c|c|}
\hline Vaccine Name & Composition & Primary type & Development phase \\
\hline MVA85A & Modified vaccinia virus Ankara expressing Ag85A & Booster vaccine & Phase 2b \\
\hline Ad5Ag85A & Recombinant adenovirus 5 expressing Ag85A & Booster vaccine & Phase 1 \\
\hline Ad35 & Recombinant adenovirus 35 expressing Ag85A-Ag85B-TB10.4 fusion protein & Booster vaccine & Phase 1/2 \\
\hline
\end{tabular}

B) Protein adjuvant booster vaccines

\begin{tabular}{|c|c|c|c|}
\hline Vaccine Name & Composition & Primary type & Development phase \\
\hline H1/IC31 & ESAT6-Ag85B fusion protein + IC31 adjuvant & Booster vaccine & Phase 1 \\
\hline HyVac4 & TB10.4-Ag85B fusion protein + IC31 adjuvant & Booster vaccine & Phase 1/2 \\
\hline ID93/GLA-SE & Rv2608-Rv3619-Rv3620-Rv1813 fusion protein + GLA-SE adjuvant & Booster vaccine & Phase 1 \\
\hline H56/IC31 & Ag85B-ESAT6-Rv2660c fusion protein + IC31 adjuvant & Booster vaccine & Phase1/2a \\
\hline M72/AS01E & Mtb39a-Mtb32a fusion protein + AS01E adjuvant & Booster vaccine & Phase 2 \\
\hline
\end{tabular}

C) Priming vaccines

\begin{tabular}{|c|c|c|c|}
\hline Vaccine Name & Composition & Primary type & Development phase \\
\hline VPM1002 & Recombinant BCG strain & Priming vaccine & Phase 2 \\
\hline MTBVAC & Live-attenuated Mycobacterium tuberculosis & Priming vaccine & Phase 1 \\
\hline
\end{tabular}

D) Therapeutic vaccines

\begin{tabular}{|c|c|c|c|}
\hline Vaccine Name & Composition & Primary type & Development phase \\
\hline RUTI $^{(B)}$ & Detoxified liposomal fragments of Mycobacterium tuberculosis & Therapeutic vaccine & Phase 2 \\
\hline Mycobacterium vaccae & Whole inactivated Mycobacterium vaccae & Therapeutic vaccine & Phase 3 \\
\hline
\end{tabular}

Based on a case-control descriptive study which has been conducted in Tehran province from 2001 to 2003, BCG vaccine efficacy and protection rate against pulmonary TB were

As indicated, there are several candidates in different development phases; however, it should not be forgotten that primary immunogenic response in animal models cannot necessarily lead to an effective vaccine in humans, as seen for MVA85A [53]. According to the WHO reports, it is expected that one or two of these candidates would enter phase 3 of the clinical trials within the next two to three years and only one vaccine would be approved to be used in 2020 [6,54].

On the other hand, the investments on production of new vaccines represent a small percentage of the disease control funds. From the amount of $\$ 46.7$ billion which was allocated by WHO for TB control between 2011 and 2015, only 4\% (\$1.9 billion) had been considered for the production of new vaccines. It is noteworthy that despite the reduction of investments between 2009 and 2010 on research and development of TB control, the amounts of investments on the control of the disease was increased from $\$ 363$ million in 2005 to $\$ 630$ million in 2012. However, this increase was not sufficient at all, since between 2013 and 2015, it is estimated that $\$ 8$ billion was required for control and treatment of TB in the low- and middle-income countries [6, 54].

TB in Iran

According to the official report of Iran's Ministry of Health, the incidence rate of all TB forms decreased from 142 cases per 100,000 in 1964 to 14 cases per 100,000 in 2015; however, it must be considered that $71 \%$ of TB cases are pulmonary ones [55]. A cross-sectional study has shown that the trend of TB smear positive incidence rate, relapses cases and mortality have been reduced in Iran from 2001 to 2008. Although successes in case detection of the disease have been reported $(2.5 \%)$, the average annual percentage change (AAPC) of TB treatment has decreased by $0.5 \%$ during 2001-2008. The trends of TB cases are higher in both sexes over 65 years of age [56]. reported to be $57 \%$ and $20 \%$, respectively [57]. On the other hand, the results of a prediction study (using Box-Jenkins models) show an increasing trend of TB cases in Iran [58]. Considering the fact that Iran has long borders with Afghanistan and Pakistan which are regarded as high-burden countries for TB in the Eastern Mediterranean Region, in addition to the low efficacy of BCG vaccine, increasing trend of TB cases in Iran, especially in over 65 year-old people and the ageing population $(10.5 \%$ and $21.7 \%$ of total population in 2020 and 2050, respectively) [59], call for an urgent and comprehensive program for prevention and control of TB in this country.

\section{Global Context of BCG Vaccination}

The Department of Immunization, Vaccines and Biologicals (IVB) of the WHO has initiated a call for nominations of experts to serve on a SAGE Working Group on the use of BCG vaccine [60]. This Working Group will be asked to review the scientific evidence in order to perform a revision of the global policy on the use of BCG. The following elements must be reviewed:

- Country practices in the use of BCG including that of targeted vaccination in low TB prevalence countries as well as the threshold applied to decide on stopping the vaccination

- TB epidemiology as well as the epidemiology of leprosy

- Trends in antibiotic resistance and their implications for BCG use

- The safety, effectiveness and duration of the protection afforded by the administration of BCG (in a strain specific analysis) in different age groups and according to HIV status and for different outcomes (i.e. death, pulmonary disease and infection) and 
assessment of the effect of booster doses (including a comparison of the effect of BCG booster/revaccination with alternative protection (e.g. isoniazid preventive therapy)

- The effects of BCG co-administration with other vaccines administered at birth (e.g. OPV and hepatitis B) or later in life (e.g. co-administration with DTP containing vaccines and specifically pentavalent vaccine)

- The economic burden of TB and cost-effectiveness of vaccination as well as modeling data to inform BCG vaccination strategies (including vaccination in the context of other control strategies)

- The potential role of BCG in the control of leprosy In addition, the Working Group will be briefed on the TB vaccine candidates' development status and will consider BCG improvement strategies that may have implications for beneficial non-specific vaccine effects of the current BCG.

\section{CONCLUSION}

TB mortality due to several complex medical and social factors is increasing worldwide and now the disease is a global issue which needs urgent attention. For this reason, a global reaction in order to prevent and control TB is deemed necessary. Unfortunately, the control and treatment of TB are very complicated due to many reasons which can be listed as poor diagnosis, failure to comply with the standard treatment protocols, multidrug-resistant strains, poverty and demographic changes, the global HIV/AIDS pandemic, lack of supervision and monitoring of full course treatments in patients, incomplete follow-up of at-risk-people and improper planning. On the other hand, BCG vaccine has a limited duration of protection and does not play a role for TB prevention and especially, pulmonary $\mathrm{TB}$ in adults. Hence, instead of attempts to manufacture new effective vaccines, it seems that more priority should be given to other TB control and prevention efforts. Some of these efforts include finding new diagnosis techniques for early detection, proper treatment and monitoring of detected cases of multidrug-resistant strains of TB, investigation for new drugs, full access to services related to TB control programs, early detection of AIDS as well as quarantining and isolation of suspicious cases at the international borders. These activities are not fruitful unless an overall TB control strategy is planned which should include written policies and procedures, appropriate laws and adequate staff, funding, networks and coalitions with the community groups to assist with implementation of TB control programs and providing a basis for evaluating the progress towards the program targets.

\section{REFERENCES}

1. Selgelid MJ. Ethics, tuberculosis and globalization. Public Health Ethics. 2008;1(1):10-20. doi:10.1093/phe/phn001.

2. Millet JP, Moreno A, Fina L, delBaño L, Orcau A, de Olalla PG et al. Factors that influence current tuberculosis epidemiology. Eur Spine J. 2013;22(Suppl.4):539-48. doi:10.1007/s00586-012-2334-8.

3. Narasimhan P, Wood J, MacIntyre CR, Mathai D. Risk Factors for Tuberculosis. Pulm Med. 2013;ArticleID:828939.11pages. doi: $10.1155 / 2013 / 828939$

4. Tsara V, Serasli E, Christaki P. Problems in diagnosis and treatment of tuberculosis infection. Hippokratia. 2009;13(1):20-2.

5. Nema V. Tuberculosis diagnostics: Challenges and opportunities. Lung India. 2012;29(3):259-66. doi:10.4103/0970-2113.99112.

6. Anderson L, Dean A, Falzon D, Floyd K, Baena IG, Gilpin C et al.
Global tuberculosis report 2015. 20th ed. World Health Organization. 2015. France.

7. Jamison DT, Breman JG, Measham AR, Alleyne G, Claeson M, Evans DB et al. Disease control priorities in developing countries. 2nd ed. Oxford University Press. 2006. USA.

8. Arnadottir T. Tuberculosis and public health. Policy and principles in tuberculosis control. International union against tuberculosis and lung disease (The Union). 2009. France.

9. Ogunremi T, Menzies D, Embil J. Prevention and control of tuberculosis transmission in health care and other settings. Canadian Tuberculosis Standard. 7th ed. Public Health Agency of Canada. 2014. Canada.

10. Dasgupta K, Menzies D. Cost-effectiveness of tuberculosis control strategies among immigrants and refugees. Eur Respir J. 2005;25:1107-16. doi:10.1183/09031936.05.00074004.

11. Okoh AC. Prevention and control of tuberculosis. Benin J Postgrad Med. 2009;11:97-9.

12. BC strategic plan for tuberculosis prevention, treatment and control. BC Communicable Disease Policy Advisory Committee. 2012; Canada.

13. Fine PEM, CarneiroIAM, Milstein JB, Clements CJ. Issues relating to the use of BCG in immunization programmes. World Health Organization. 1999. Switzerland.

14. Kernodle DS. Decrease in the effectiveness of Bacille Calmette-Guérin vaccine against pulmonary tuberculosis: a consequence of increased immune suppression by microbial antioxidants, not overattenuation. Clin Infect Dis. 2010;51(2):177-84.

15. Wittes RC. Immunology of Bacille Calmette-Guérin and related topics. Clin Infect Dis. 2000;31(Suppl.3):S59-S63.

16. Setia MS, Steinmaus C, Ho CS, Rutherford GW. The role of BCG in prevention of leprosy: a meta-analysis. Lancet Infect Dis. 2006;6(3):162-70. doi:10.1016/S1473-3099(06)70412-1.

17. Merle CS, Cunha SS, Rodrigues LC. BCG vaccination and leprosy protection: review of current evidence and status of BCG in leprosy control. Expert Rev Vaccines. 2010;9(2):209-22. doi:10.1586/ERV.09.161.

18. Tanghe A, Content J, Van Vooren JP, Portaels F, Huygen K. Protective efficacy of a DNA vaccine encoding antigen 85A from Mycobacterium bovis BCG against Buruli ulcer. Infect Immun. 2001;69(9):5403-11. doi:10.1128/IAI.69.9.5403-5411.2001.

19. Lamm DL, Blumenstein BA, Crawford ED, Montie JE, Scardino P, Grossman $\mathrm{HB}$ et al. A randomized trial of intravesical doxorubicin and immunotherapy with bacille Calmette- Guérin for transitional-cell carcinoma of the bladder. N Engl J Med. 1991;325(2):1205-9. doi:10.1056/NEJM199110243251703.

20. Mosolits S, Nilsson B, Mellstedt H. Towards therapeutic vaccines for colorectal carcinoma: a review of clinical trials. Expert Rev Vaccines. 2005;4(3):329-50. doi:10.1586/14760584.4.3.329.

21. Miyazawa N, Suemasu K, Ogata T, Yoneyama T, Naruke T, Tsuchiya R. BCG immunotherapy as an adjuvant to surgery in lung cancer: a randomized prospective clinical trial. Jpn J Clin Oncol. 1979;9(1):19-26.

22. Sari A, Bal K, Tunakan M, Ozturk C. A case of a sporadic malignant peripheral nerve sheath tumor of the urinary bladder with concomitant in situ urothelial carcinoma treated by transuretheral resection. Indian $\mathrm{J}$ PatholMicrobiol. 2011;54(1):147-9.

23. Krone B, Kölmel KF, Henz BM, Grange JM. Protection against melanoma by vaccination with Bacille Calmette-Guérin (BCG) and/or vaccinia: an epidemiology-based hypothesis on the nature of a melanoma risk factor and its immunological control. Eur J Cancer. 2005;41(1):104-17. 24. Paolillo A, Buzzi MG, Giugni E, Sabatini U, Bastianello S, Pozzilli C et al. The effect of Bacille Calmette-Guérin on the evolution of new enhancing lesions to hypointense T1 lesions in relapsing remitting MS. J Neurol. 2003;250(2):247-8. doi:10.1007/s00415-003-0967-6.

25. Rutschmann OT, Mc Crory DC, Matchar DB. Immunization panel of the multiple sclerosis council for clinical practice guidelines. Immunization and MS: a summary of published evidence and recommendations. Neurol. 2002;59(12):1837-43.

26. Ristori G, Buzzi MG, Sabatini U, Giugni E, Bastianello S, Viselli F et al. Use of bacille Calmette-Guérin (BCG) in multiple sclerosis. Neurol. 1999;53(7):1588-9.

27. Yong J, Lacan G, Dang H, Hsieh T, Middleton B, Wasserfall C et al. BCG Vaccine-Induced neuroprotection in a mouse model of Parkinson's disease. PLoS ONE. 2011;6(1):e16610. doi:10.1371/journal.pone.0016610. 28. Laćan G, Dang H, Middleton B, Horwitz MA, Tian J, Melega WP et al. Bacillus Calmette-Guérin vaccine-mediated neuroprotection is associated with regulatory T-cell induction in the 1-methyl-4-phenyl-1,2,3,6tetrahydropyridine mouse model of Parkinson's disease. J Neurosci Res. 2013;91(10):1292-1302. doi:10.1002/jnr.23253. 
29. Chan ED, Iseman MD. Current medical treatment for tuberculosis. Brit Med J. 2002;325(7375):1282-6.

30. Venkataswamy MM, Goldberg MF, Baena AC, Chan J, Jacobs WR Jr, Porcelli SA. In vitro culture medium influences the vaccine efficacy of Mycobacterium bovis BCG. Vaccine. 2012;30(6):1038-49. doi:10.1016/j.vaccine.2011.12.044.

31. Salisbury D, Ramsay M, Noakes K. Immunisation against infectious disease. 3rd ed. Stationery office under licence from the department of health. 2006. England.

32. Colditz GA, Brewer TF, Berkey CS, Wilson ME, Burdick E, Fineberg $\mathrm{HV}$ et al. Efficacy of BCG vaccine in the prevention of tuberculosis. Metaanalysis of the published literature. J Am Med Assoc. 1994;271(9):698-702. 33. Rodrigues LC, Diwan VK, Wheeler JG. Protective effect of BCG against tuberculous meningitis and miliary tuberculosis: a meta-analysis. Int J Epidemiol. 1993;22(6):1154-8.

34. Datta M, Valishayee SRS, Diwakara SAM. Fifteen years follow up of trial of BCG vaccines in south India for tuberculosis prevention. Indian J Med Res. 1999;110:56-69.

35. Glyn Hewinson R. TB vaccines for the World. Tuberculosis. 2005;85(12):1-6. doi:10.1016/j.tube.2004.10.002.

36. Sterne JA, Rodrigues LC, Guedes IN. Does the efficacy of BCG decline with time since vaccination? Int J Tuberc Lung Dis. 1998;2(3):200-7.

37. Aronson NE, Santosham M, Comstock GW, Howard RS, Moulton LH, Rhoades ER et al. Long-term efficacy of BCG vaccine in American Indians and Alaska Natives: A 60-year follow-up study. J Am Med Ass. 2004;291(17):2086-91.

38. Pereira SM, Dantas MSD, Ximenes R, Barreto ML. BCG vaccine against tuberculosis: its protective effect and vaccination policies. Rev SaúdePública. 2007;41(Supl.1):59-66. doi:10.1590/S003489102007000800009

39. Ahmad NA, Hamid HA, Sahril N, Yusoff MF, Naidu BM, Aris T. Bacille Calmette- Guérin (BCG) Revaccination: Is it beneficial for tuberculosis control? Open Access Scientific Reports. 2013;2:656. doi: $10.4172 /$ scientificreports. 656 .

40. Brosch R, Gordon SV, Garnier T, Eiglmeier K, Frigui W, Valenti P et al. Genome plasticity of BCG and impact on vaccine efficacy. Proc Natl Acad Sci. 2007;104(13):5596-5601. doi:10.1073/pnas.0700869104.

41. Bricks LF. Percutaneous or intradermal BCG vaccine? J Pediatr (Rio J). 2004;80(2):93-8.

42. Packe GE, Innes JA. Protective effect of BCG vaccination in infant Asians: a case-control study. Arch Disease Childhood. 1988;63(3):277-81. doi:10.1136/adc.63.3.277

43. Black GF1, Weir RE, Floyd S, Bliss L, Warndorff DK, Crampin AC, et al. BCG-induced increase in interferon-gamma response to mycobacterial antigens and efficacy of BCG vaccination in Malawi and the UK: two randomized controlled studies. Lancet. 2002;359:(9315):1393-1401. doi:10.1016/S0140-6736(02)08353-8.

44. Flaherty DK, Vesosky B, Beamer GL, Stromberg P, Turner J. Exposure to Mycobacterium aviumcan modulate established immunity against Mycobacterium tuberculosis infection generated by Mycobacterium bovis BCG vaccination. J Leukoc Biol. 2006;80:1262-71.
45. Brandt L, Feino Cunha J, Olsen AW, Chilima B, Hirsch P, Appelberg R et al. Failure of Mycobacterium bovis BCG vaccine: some species of environmental mycobacteria block multiplication of BCG and induction of protective immunity to tuberculosis. Infect Immun 2002;70(2):672-8. doi:10.1128/IAI.70.2.672-678.2002.

46. Rook GAW, Dheda K, Zumla A. Do successful tuberculosis vaccines need to be immunoregulatory rather than merely Th1-boosting? Vaccine. 2005;23(17-18):2115-20. doi:10.1016/j.vaccine.2005.01.069.

47. Jeevan A, Sharma AK, McMurray DN. Ultraviolet radiation reduces resistance to Mycobacterium tuberculosis infection in BCG-vaccinated $\begin{array}{lll}\text { guinea } & \text { pigs. } & \text { Tuberculosis. }\end{array}$ doi:10.1016/j.tube.2009.09.004.

48. Okada M. Novel vaccines against M. tuberculosis. Kekkaku. 2006;81(12):745-51.

49. vonReyn CF, Vuola JM. New vaccines for the prevention of tuberculosis. Clin Inf Dis. 2002;35:465-474.

50. Martin C. Tuberculosis vaccines: past, present and future. Cur Opin Pulm Med. 2006;12(3):186-91.

51. Franco-Paredes C, Rouphael N, del Rio C, Santos-Preciado JI. Vaccination strategies to prevent tuberculosis in the new millennium: from BCG to new vaccine candidates. Int Soc Infect Dis. 2006;10:93-102.

52. Montagnani C, Chiappini E, Galli L, de Martino M. Vaccine against tuberculosis: what's new? BMC Inf Dis. 2014;14(Suppl.1):S2. doi:10.1186/1471-2334-14-S1-S2.

53. Tameris MD, Hatherill M, Landry BS, ScribaTJ, Snowden MA, Lockhart S et al. Safety and efficacy of MVA85A, a new tuberculosis vaccine, in infants previously vaccinated with BCG: a randomised, placebocontrolled phase 2b trial. Lancet. 2013;381(9871):1021-8. doi:10.1016/S0140-6736(13)60177-4.

54. Dias HM, Falzon D, Fitzpatrick C, Floyd K, Glaziou P, Hiatt T et al Global tuberculosis report 2012. World Health Organization. World Health Organization. 2012. France.

55. The incidence of TB in Iran. Official Report of Iran's Ministry of Health and Medical $\quad 2015 . \quad \mathrm{http}: / / \mathrm{tb}$ lep.behdasht.gov.ir/TB Situation in Iran.aspx.

56. ArsangSH, Kazemnejad A, Amani F. Epidemiology of tuberculosis in Iran (2001-2008). J Gorgan Uni Med Sci. 2011;13(3):78-86 [Article in Persian].

57. Alimagham M, Aminiafshar S, Farahmand S, Vahdani $\mathrm{P}$, AlaviMoghadam M, Sharafi K. BCG vaccination and active tuberculosis prevention: A three-year study. Tanaffos. 2007;6(2):63-67.

58. Moosazadeh M, Nasehi M, Bahrampour A, Khanjani N, Sharafi S, Ahmadi S. Forecasting tuberculosis incidence in Iran using Box-Jenkins models. Iran Red Crescent Med J. 2014;16(5):e11779. doi:10.5812/ircmj.11779.

59. Danial Z, Motamedi MHK, Mirhashemi S, Kazemi A, Mirhashemi AH Ageing in Iran. Lancet. 2014; 384(9958):1927. doi:10.1016/S0140 6736(14)62278-9.

60. Call for nomination for experts to serve on a SAGE working group on the use of bacilleCalmette-Guérin (BCG) vaccine. 2016. World Health Organization.

http://www.who.int/immunization/policy/sage/working_group_bcg/en/. 